\title{
Poster: Effects of Head Tracking and Stereo on Non-Isomorphic 3D Rotation
}

\author{
Joseph J. LaViola Jr.* \\ University of Central Florida \\ School of EECS \\ Orlando, FL 32816
}

\author{
Andrew S. Forsberg ${ }^{\dagger}$ \\ Brown University \\ Dept. of Computer Science \\ Providence, RI 02912
}

\author{
John Huffman \\ Brown University \\ Center for Computation and \\ Visualization \\ Providence, RI 02912
}

\author{
Andrew Bragdon $§$ \\ Brown University \\ Dept. of Computer Science \\ Providence, RI 02912
}

\begin{abstract}
We present an experimental study that explores how head tracking and stereo affect user performance when rotating 3D virtual objects using isomorphic and non-isomorphic rotation techniques. Our experiment compares isomorphic with non-isomorphic rotation utilizing four different display modes (no head tracking/no stereo, head tracking/no stereo, no head tracking/stereo, and head tracking/stereo) and two different angular error thresholds for task completion. Our results indicate that rotation error is significantly reduced when subjects perform the task using non-isomorphic 3D rotation with head tracking/stereo than with no head tracking/no stereo. In addition, subjects performed the rotation task with significantly less error with head tracking/stereo and no head tracking/stereo than with no head tracking/no stereo, regardless of rotation technique. Subjects also highly rated the importance of stereo and non-isomorphic amplification in the 3D rotation task.
\end{abstract}

Keywords: non-isomorphic 3D rotation, stereo, head tracking, evaluation

Index Terms: H.5.2 [Information Interfaces and Presentation]: User Interfaces—Evaluation/methodology

\section{INTRODUCTION}

Effectively rotating objects in 3D space is an important part of many 3D user interfaces. In fact, rotating 3D objects is part of one of the fundamental 3D interaction tasks (i.e., selection and manipulation) used in 3D applications [1]. Given this task is a common component of many $3 \mathrm{D}$ user interfaces, it is important to evaluate and understand how 3D rotation techniques perform under different conditions so guidelines can be established. These guidelines can then assist 3D user interface designers in choosing appropriate $3 \mathrm{D}$ rotation techniques that maximize speed and efficiency while minimizing rotational error.

One approach to rotating objects in 3D space is to use nonisomorphic mappings [1]. Non-isomorphic mappings let users interact with virtual world objects at an amplified scale, in contrast to isomorphic mappings (i.e., one-to-one mappings) that maintain a direct correspondence with the physical and virtual worlds. Although there has been work on assessing the effectiveness of nonisomorphic 3D rotation $[3,4]$ in both conventional desktop and immersive virtual environments (VEs), the specific effects of stereoscopic viewing and head tracking on non-isomorphic 3D rotation has not been rigorously explored. Thus, we present a usability study that explores how user performance with both isomorphic and nonisomorphic 3D rotation is affected by different VE display modes

\footnotetext{
*e-mail: jjl@cs.ucf.edu

†e-mail: asf@cs.brown.edu

†e-mail:John_Huffman@Brown.edu

§email: Andrew_Bragdon@Brown.edu
}

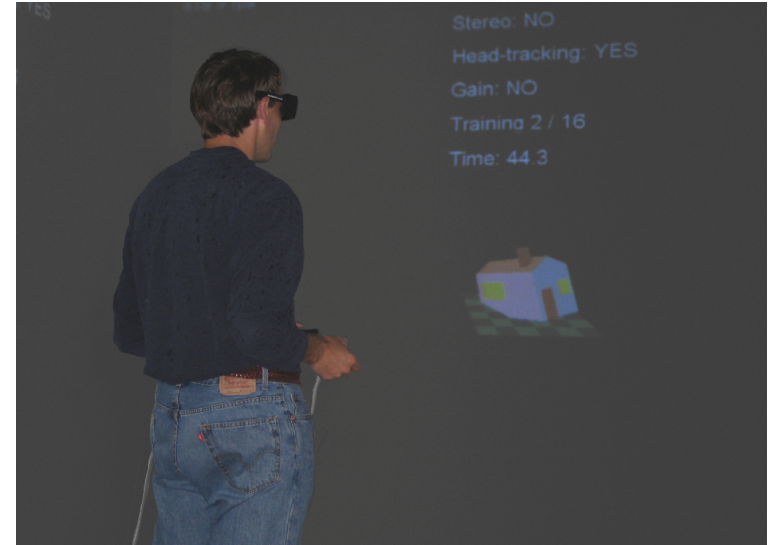

Figure 1: A user rotating the house model to its target orientation.

(no head tracking/stereo, stereo only, head tracking only, both head tracking and stereo).

\section{EXPERIMENTAL Study}

We conducted an experimental study to explore how head tracking and stereoscopic vision impact user performance when rotating virtual objects using isomorphic and non-isomorphic 3D rotation techniques.

\subsection{Subjects and Apparatus}

Sixteen subjects ( 9 male, 7 female) were recruited from the Brown University population with ages ranging from 18 to 50 . Of the 16 subjects, 14 were right handed and two were left handed. 11 subjects had little or no experience with 6DOF input devices.

The experiments were conducted in Brown University's surround screen virtual environment (three walls and a floor) at a resolution of $1024 \times 768$ per wall. The refresh rate was $120 \mathrm{~Hz}(60 \mathrm{~Hz}$ per eye). A 6DOF Polhemus FASTRAK magnetic sensor was placed inside a rubber ball and used as the input device for rotating the virtual objects. A Wanda (i.e., 6DOF joystick) was used as a triggering device in the non-dominant hand.

\subsection{Experimental Task}

Participants were instructed to rotate a solid shaded 3D model of a house from a randomly generated orientation into a target orientation (see Figure 1), a task that has been used in several studies in the past $[3,4]$. The house was designed to provide maximum cues to understanding its orientation from any angle, with asymmetric placement of windows, its chimney, and the coloring of its walls.

Users could rotate the house when the button on the Wanda was depressed. The user would start or stop the rotation by pressing or releasing the button on the Wanda. The user could iteratively rotate the house by holding the button, rotating the ball device, releasing the button, repositioning the ball device, holding the button, etc. as 
many times as necessary. When the orientation error was below a threshold, the house would immediately disappear and reappear in a new random orientation, indicating that the trial had been accomplished.

\subsection{Experiment Design and Procedure}

We used a $4 \times 2 \times 2$ balanced, within subjects factorial design where the independent variables were display mode (i.e., the four permutations of head-tracking and stereo on or off), rotation amplification, and the orientation error threshold. The coefficient of amplification varied between either one (isomorphic rotation) or three (nonisomorphic rotation). The orientation error threshold was either six or 18 degrees. We used the same set of 10 random house rotations each of which had error between 70 and 180 degrees. Each subject completed 10 repetitions of each of the 16 conditions for a total of 160 trials.

The dependent variables were task completion time, and final orientation error. Completion time is the time from the user first pressing the Wanda button until releasing the button while the orientation error is below the error threshold. Orientation error is the angular distance between the orientation of the house upon completing a trial and the house's target orientation.

The experiments began with a pre-questionnaire, followed by an explanation of the SSVE, the devices involved, the experimental task and procedure, and the techniques involved in accomplishing the task. There was then a training session where the subject was given one trial under each of the 16 conditions to be tested (each possible combination of four display modes, two amplification coefficients and two error thresholds). In a post-questionnaire, subjects were asked how important head tracking, stereo, and rotation amplification was in completing the rotation task. A five point Lickert scale was used (very low, low, moderate, high, and very high).

\subsection{Results}

A repeated measures three-way analysis of variance (ANOVA) was performed for each of the dependent variables with display mode (DM), amplification coefficient (AC), and error threshold (ET) as the independent variables. Table 1 summarizes the main effects of the independent variables. Display mode, amplification coefficient, and error threshold significantly affected both task completion time and error. There were no significant interaction effects between the three independent variables.

\begin{tabular}{|c|c|c|}
\hline Effect & Time & Error \\
\hline \multirow{2}{*}{$\mathrm{DM}$} & $\begin{array}{c}F_{3,13}=3.33 \\
p<0.05\end{array}$ & $\begin{array}{c}F_{1.79,13}=8.193 \\
p<0.05\end{array}$ \\
\hline \multirow{2}{*}{$\mathrm{AC}$} & $\begin{array}{c}F_{1,15}=4.68 \\
p<0.05\end{array}$ & $\begin{array}{c}F_{1,15}=13.8 \\
p<0.05\end{array}$ \\
\hline \multirow{2}{*}{$\mathrm{ET}$} & $\begin{array}{c}F_{1,15}=11.77 \\
p<0.05\end{array}$ & $\begin{array}{c}F_{1,15}=15.46 \\
p<0.05\end{array}$ \\
\hline
\end{tabular}

Table 1: The main effects for display mode (DM), amplification coefficient (AC), and error threshold (ET) for both time and error. Note that for error under DM, the sphericity assumption was violated resulting in a Greenhouse-Geisser correction.

We conducted a post-hoc analysis on display mode (DM) for both completion time and error to gain a better understanding of its effect on user performance in our rotation task. We performed pairwise comparisons using Holm's sequential Bonferroni adjustment [2] with six comparisons for each dependent variable at $\alpha=0.05$ for no head tracking/no stereo (DM1), no head tracking/stereo (DM2), head tracking/no stereo (DM3), and head tracking/stereo (DM4).

For task completion time, subjects completed the rotation task significantly faster $\left(t_{15}=-3.149, p<0.0083\right)$ under the no head tracking/stereo (DM2) mode (4.25 seconds) than with the head tracking/stereo (DM4) mode (4.6 seconds). All other pairwise comparisons were not significant.

For error, subjects completed the rotation task with significantly less error $\left(t_{15}=4.4, p<0.0083\right)$ under the head tracking/stereo (DM4) mode (3.63 degrees) than with the head tracking/no stereo (DM3) mode (4.11 degrees) and with significantly less error $\left(t_{15}=\right.$ $3.39, p<0.0125$ ) than with the no head tracking/no stereo (DM1) mode (4.39 degrees). In addition, subjects also performed with significantly less error $\left(t_{15}=3.47, p<0.01\right)$ under the no head tracking/stereo (DM2) mode (3.92 degrees) than with no head tracking/no stereo (DM1). All other pairwise comparisons were not significant. These results indicate that stereo plays an important role in user performance when performing 3D rotation tasks.

We also conducted a post-hoc analysis on the amplification coefficients (AC) in each display mode (DM) for both completion time and error. We performed pairwise comparisons using Holm's sequential Bonferroni adjustment [2] with 10 comparisons for each dependent variable at $\alpha=0.05$. Four comparisons are used to test for significance between isomorphic rotation $(\mathrm{AC} 1)$ and nonisomorphic rotation (AC3) within each display mode with the remaining six comparisons testing for significance between $\mathrm{AC} 1$ and AC3 across display modes.

For task completion time, we did not find any significant differences for the 10 pairwise comparisons due to the Bonferroni correction. However, for error, we found that subjects were significantly more accurate $\left(t_{15}=3.487, p<0.005\right)$ when using the non-isomorphic technique under the head tracking/stereo display (DM4) mode (3.75 degrees) than under the no head tracking/no stereo display (DM1) mode (4.52 degrees). This result indicates that the combination of head tracking and stereo plays an important role in a subject's ability to accurately perform non-isomorphic rotation tasks.

In addition to the dependent variables in our experiment, subjects filled out a post-questionnaire and were asked to rank, using a five point Lickert scale, how important they felt stereo, head tracking, and rotation amplification affected there ability to perform the orientation matching task. The importance rankings indicate that subjects felt stereo was moderately to highly important in completing the rotation task, while amplification factor seemed to be very important. The results where mixed on the importance of head tracking in the experiment as the rankings were fairly well spread over the five point scale in a normally distributed fashion.

\section{CONCLUSION}

We have presented an experiment which explores how stereoscopic viewing and head tracking affect user performance when using isomorphic and non-isomorphic rotation to complete 3D orientation matching tasks. Our results indicate that stereo plays an important role in rotation accuracy when performing 3D rotation tasks, regardless of rotation technique, and that both head tracking and stereo are important to rotation accuracy when non-isomorphic rotation is used. In addition, subjects highly rated the importance of stereo and non-isomorphic amplification in the 3D rotation task.

\section{REFERENCES}

[1] D. Bowman, E. Kruijff, J. LaViola, and I. Poupyrev. 3D User Interfaces: Theory and Practice. Addison-Wesley, 2004.

[2] S. Holm. A simple sequentially rejective multiple test procedure. Scandinavian Journal of Statistics, 6:60-65, 1979.

[3] J. J. LaViola and M. Katzourin. An exploration of non-isomorphic 3d rotation in surround screen virtual environments. In Proceedings of the IEEE Symposium on 3D User Interfaces 2007, pages 49-54, 2007.

[4] I. Poupyrev, S. Weghorst, and S. Fels. Non-isomorphic 3d rotational techniques. In CHI '00: Proceedings of the SIGCHI Conference on Human Factors in Computing Systems, pages 540-547, New York, NY, USA, 2000. ACM Press. 